\title{
Combined catecholamine and indoleamine depletion following response to ECT
}

\author{
Frederick Cassidy, Richard D. Weiner, Thomas B. Cooper and Bernard J. Carroll
}

\section{Summary}

The mechanism of action of electroconvulsive therapy (ECT) in treating major depression is unknown. We studied two candidate mechanisms through inhibiting simultaneously the synthesis of noradrenaline and serotonin in patients immediately after successful treatment with ECT using a randomised, placebo-controlled, double-blind crossover design. There were no significant changes in depression scores under any experimental conditions, or between the amine-depleted and placebo groups despite reductions of $61 \%$ in serum homovanillic acid, $47 \%$ in 3-methoxy-4hydroxyenylethyleneglycol, and $89 \%$ in serum tryptophan. Catecholamine and serotonin availability may not be necessary for maintaining the initial antidepressant response to ECT.

\section{Declaration of interest}

None.
The mechanism of action of electroconvulsive therapy (ECT) remains unclear despite its status as a highly effective treatment for major depressive episodes. Studies of ECT have focused on the serotonergic and noradrenergic systems, ${ }^{1}$ but without conclusive findings. Two probes explored in studying mechanisms of antidepressant drug response are indoleamine depletion ${ }^{2}$ and catecholamine depletion. ${ }^{3}$ Individuals with depression treated successfully with noradrenergic antidepressants tended to transiently relapse with catecholamine depletion, whereas those treated successfully with serotonergic antidepressants tended to transiently relapse with indoleamine depletion. We have shown that neither indoleamine depletion nor catecholamine depletion, given alone, led to signs of relapse in individuals soon after successful treatment with ECT. ${ }^{4,5}$

It is possible that ECT operates through multiple neurotransmitter systems. Moreover, these neurotransmitter systems are known to interact with each other. For example, a compensatory increase in serotonergic function may have attenuated or blocked a response to catecholamine depletion, ${ }^{6}$ particularly if the mechanism of ECT were to relate to both systems. We therefore conducted a double catecholamine and indoleamine depletion study in individuals with depression.

\section{Method}

Seven male and two female in-patients (mean age 50.4 years (s.d.=11.3), range 30-70) initially meeting DSM-IV criteria for major depressive episode, ${ }^{7}$ four with psychotic features, and who had responded to courses of bilateral ECT were included. Each gave written informed consent for participation in the study, which was approved by both the institutional review board at Duke University Medical Center and the research committee at John Umstead Hospital. The mean Montgomery-Åsberg Depression Rating Scale $(\mathrm{MADRS})^{8}$ score prior to ECT was 35.0 (s.d. $=5.4$, range 26-43); ECT was administered as described previously ${ }^{5}$ (mean number of treatments received 10.4 , s.d. $=3.9$, range $7-18)$.

The study used a double-blind, counterbalanced, placebocontrolled design over two 4-day periods commencing after the next to last and the last ECT treatments. In each 4-day period, Day 1 was designated pre-baseline. Day 2 was designated baseline: the catecholamine depletion protocol was commenced. On Day 3 , the catecholamine depletion protocol was continued, and indoleamine depletion was added to achieve combined depletion. The definitive behavioural and biochemical assessments were performed at $15.00 \mathrm{~h}$ on Day 3. Day 4 was designated a followup day. Consistent with clinical practice when the study was performed, participants remained off psychotropic medication throughout the ECT treatments, and throughout this study.

Participants were evaluated at $09.00 \mathrm{~h}$ each day with the MADRS. Stability of MADRS scores from Day 1 to Day 2 was required. Serum was collected on Day 2 (baseline) at $09.00 \mathrm{~h}$ for measurement of homovanillic acid (HVA), 3-methoxy-4hydroxyenylethyleneglycol (MHPG) and tryptophan levels. Catecholamine depletion was effected with alpha-methyl-ptyrosine (AMPT) $1 \mathrm{~g}$ orally at $09.00 \mathrm{~h}, 12.00 \mathrm{~h}$ and $15.00 \mathrm{~h}$ on Day 2, and at $09.00 \mathrm{~h}$ and $12.00 \mathrm{~h}$ on Day 3. For the placebo condition, we gave $50 \mathrm{mg}$ diphenhydramine as an active placebo at the same times to mask the sedation associated with AMPT. On Day 3, serum was obtained again at $09.00 \mathrm{~h}$ for measurement of HVA, MHPG and tryptophan levels. To achieve indoleamine depletion, individuals who had received active AMPT were given a full-strength tryptophan-free amino acid mixture ${ }^{9}$ at $09.00 \mathrm{~h}$ on Day 3. Participants who had received the active placebo were given a quarter-strength tryptophan-free amino acid mixture to preserve masking. To further limit tryptophan availability, individuals fasted on Day 3 until after the completion of the afternoon ratings. At $03.00 \mathrm{~h}$ on Day 3, MADRS ratings were completed to document the primary end-point, and serum was again obtained. Within the next 3 days, participants received their final ECT treatment, and the study was repeated with the alternate active or placebo protocol. They were given a low monoamine diet during the study. Tryptophan was assayed by high-performance liquid chromatography, and HVA and MHPG were assayed as described elsewhere. ${ }^{10}$ Insufficient serum was available for three of the MHPG samples.

Changes in MADRS scores at depletion end-point $(15.00 \mathrm{~h}$ on Day 3) compared with post-ECT baseline ( $09.00 \mathrm{~h}$ on Day 2$)$ were analysed to compare the effect of combined catecholamine and indoleamine depletion with the effect of the placebo protocol on mood state using $t$-tests. Each individual served as their own control. Two participants who completed only the active arms of the study were excluded from this analysis. Their MADRS scores at baseline and at the depletion end-point were 1 and 1 respectively for the one person, and 12 and 13 for the other.

\section{Results}

Mean post-ECT baseline MADRS scores (7.0, s.d.=4.4) were reduced in comparison to pre-ECT scores (mean $=35.0$, s.d. $=5.4, t=10.7$, d.f. $=8, P<0.001)$. Following the administration of AMPT and the full-strength amino acid load, mean serum HVA levels decreased $60.8 \%$ and MHPG level decreased $46.6 \%$ 
Table 1 Mean Montgomery-Åsperg Depression Rating Scale (MADRS) scores and biochemical assessments during depletion and active placebo

\begin{tabular}{|c|c|c|c|c|c|}
\hline & \multicolumn{3}{|c|}{ Baseline } & \multirow{2}{*}{$\begin{array}{c}\text { End point } \\
\text { Day } 3,15.00 \mathrm{~h}\end{array}$} & \multirow{2}{*}{$\begin{array}{c}\text { Follow-up } \\
\text { Day } 4,09.00 \mathrm{~h}\end{array}$} \\
\hline & Day $1,09.00 \mathrm{~h}$ & Day $2,09.00 \mathrm{~h}$ & Day $3,09.00 \mathrm{~h}$ & & \\
\hline \multicolumn{6}{|c|}{ Combined monoamine depletion, mean (s.d.) $(n=9)$} \\
\hline MADRS score & $7.4(4.5)$ & $6.9(5.5)$ & $6.9(4.8)$ & $6.6(4.3)$ & $5.8(4.4)$ \\
\hline Serum HVA, ng/ml & & $17.6(6.2)$ & $5.8(1.9)$ & $6.9(1.6)$ & \\
\hline Serum MHPG, ng/ml & & $4.5(3.1)$ & $1.5(0.4)$ & $2.4(1.2)$ & \\
\hline Serum tryptophan, $\mu g m / m l$ & & $11.5(2.8)$ & $9.8(2.2)$ & $1.1(0.7)$ & \\
\hline \multicolumn{6}{|c|}{ Placebo control protocol, mean (s.d.) $(n=7)$} \\
\hline MADRS score & $6.0(3.9)$ & $7.1(5.3)$ & $4.9(4.1)$ & $5.7(4.9)$ & $6.7(6.6)$ \\
\hline Serum HVA, ng/ml & & $15.7(5.1)$ & $15.1(5.6)$ & $14.9(5.2)$ & \\
\hline Serum MHPG, ng/ml & & $3.4(0.9)$ & $4.5(3.0)$ & $5.1(1.7)$ & \\
\hline Serum tryptophan, $\mu g m / m l$ & & $11.7(3.4)$ & $10.6(3.3)$ & $5.9(2.6)$ & \\
\hline
\end{tabular}

from baseline to the combined depletion end-point, while the mean serum tryptophan level drawn immediately prior to the active $100 \%$ amino acid load decreased $88.8 \%$ at the combined depletion end-point (Table 1). A substantial 67\% decrease in both mean serum HVA levels and mean serum MHPG were observed following 24 hours of AMPT alone (Day 3, $09.00 \mathrm{~h}$ ), compared with mean levels at baseline (Day 2, $9.00 \mathrm{~h}$; Table 1). No clinical changes were noted during the combined catecholamine/ indoleamine depletion in any individual. Pairs of MADRS scores at baseline and at the depletion end-point during active depletion were: 5,$6 ; 3,4 ; 14,13 ; 0,2 ; 8,5 ; 5,6 ; 11,9 ; 1,1$; and 12,13 . Change in MADRS scores during depletion were not significant $(t=0.755$ d.f. $=12,95 \%$ CI 3.477 to -0.905$)$. Analysis of MADRS scores also indicated neither period $(t=2.012$, d.f. $=12,95 \%$ CI 3.915 to -0.201$)$ nor carry-over $(t=1.779$, d.f. $=12,95 \%$ CI 2.862 to -3.528) effects. Secondary analyses of change in MADRS scores from baseline (Day 2, $9.00 \mathrm{~h}$ ) following 1 day of AMPT or active placebo (Day 3, $9.00 \mathrm{~h}$ ) revealed no significant differences between experimental interventions $(t=0.988$, d.f. $=12,95 \%$ CI 5.9826 to -1.4106$)$. Neither period $(t=1.329$, d.f. $=12,95 \%$ CI 6.208 to -1.064$)$ nor carry-over $(t=1.003$, d.f. $=12,95 \%$ CI 6.406 to $-7.906)$ effects were observed.

\section{Discussion}

Combined functional blockade of catecholaminergic and serotonergic systems had no effect on maintenance of an acute response to ECT. This result augments our findings from single blockade of these systems. Viewed collectively, these studies do not support a major role of catecholamine or serotonin, either alone or through a synergistic mechanism, in maintaining the early response to ECT in major depression. The combined depletion strategy makes it unlikely that homeostatic compensatory effects between noradrenergic and serotonergic systems prevented relapses in our earlier studies of single amine depletion. Although sample size was limited in this study, the mean rating scores (Table 1) suggest that if a statistically significant difference were noted between combined amine depletion and the placebo protocol, the magnitude of the effect would be clinically trivial.

Our findings bear on the issue of relapse after successful ECT. Despite the efficacy of ECT in major depression, relapse rates remain unacceptably high, even with the use of maintenance ECT. Relapse rates about 24-28 weeks following ECT for a depressive episode have been reported at $80-84 \%$ on no prophylactic medication, ${ }^{11} 60 \%$ on lithium alone, $37-39 \%$ on lithium and nortriptyline, ${ }^{11,12}$ and $37 \%$ with maintenance ECT. ${ }^{12}$ Our results suggest that the classical catecholamine and indoleamine neurotransmitter systems are not of primary importance for maintaining an acute response to ECT. New approaches to maintenance therapy are, therefore, needed.
Frederick Cassidy, MD, Department of Psychiatry and Behavioral Sciences, Duke University, Durham, and Central Regional Hospital, Butner, North Carolina; Richard D. Weiner, MD, PhD, Department of Psychiatry and Behavioral Sciences, Duke University, Durham, amd Veteran's Administration Hospital, Durham, North Carolina; Thomas B. Cooper, MA, The Nathan S. Kline Institute for Psychiatric Research, Orangeburg, New York; Bernard J. Carroll, MB, PhD, Department of Psychiatry and Behavioral Sciences, Duke University, Durham, North Carolina, and The Pacific Behavioral Research Foundation, Carmel, California, USA

Correspondence: Dr F. Cassidy, Central Regional Hospital, 300 Veazy Street, Butner, NC 27509, USA. Email: cassi002@mc.duke.edu

First received 23 Sep 2009, final revision 11 Jan 2010, accepted 11 Feb 2010

\section{Funding}

This project was supported by a NARSAD young investigator award.

\section{References}

1 Fochtmann LJ. Animal studies of electroconvulsive therapy: foundations for future research. Psychopharmacol Bull 1994; 30: 321-444.

2 Delgado PL, Charney DS, Price LH, Aghajanian GK, Landis H, Heninger GR. Serotonin function and the mechanism of antidepressant action. Reversal of antidepressant-induced remission by rapid depletion of plasma tryptophan. Arch Gen Psychiatry 1990; 47: 411-8.

3 Miller $\mathrm{HL}$, Delgado PL, Salomon RM, Berman R, Krystal JH, Heninger GR, et al. Clinical and biochemical effects of catecholamine depletion on antidepressant-induced remission of depression. Arch Gen Psychiatry 1996; 53: $117-28$.

4 Cassidy F, Weiner RD, Cooper TB, Carroll BJ. Antidepressant response to electroconvulsive therapy is sustained after catecholamine depletion. Prog Neuropsychopharmacol Biol Psychiatry 2009; 33: 872-4.

5 Cassidy F, Murry E, Weiner RD, Carroll BJ. Lack of relapse with tryptophan depletion following successful treatment with ECT. Am J Psychiatry 1997; 154: 1151-2.

6 Gobert A, Rivet JM, Audinot V, Newman-Tancredi A, Cistarelli L, Millan MJ. Simultaneous quantification of serotonin, dopamine and noradrenaline levels in single frontal cortex dialysates of freely-moving rats reveals a complex pattern of reciprocal auto- and heroreceptor-mediated control of release. Neuroscience 1998; 84: 413-29.

7 American Psychiatric Association. Diagnostic and Statistical Manual of Mental Disorders (4th edn) (DSM-IV). APA, 1994.

8 Montgomery SA, Åsberg M. A new depression scale designed to be sensitive to change. Br J Psychiatry 1979; 134: 382-9.

9 Cassidy F, Murry E, Carroll BJ. Tryptophan depletion in recently manic patients treated with lithium. Biol Psychiatry 1998; 43: 230-2.

10 Fri CG, Wiesel FA, Sedvall G. Simultaneous quantification of homovanillic acid and 5-hydroxyindoleacetic acid in cerebrospinal fluid by mass fragmentography. Life Sci 1974; 14: 2469-80.

11 Sackeim HA, Haskett RF, Mulsant BH, Thase ME, Mann JJ, Pettinati HM, et al. Continuation pharmacotherapy in the prevention of relapse following electroconvulsive therapy: a randomized controlled trial. JAMA 2001; 285: 1299-307.

12 Kellner CH, Knapp RG, Petrides G, Rummans TA, Husain MM, Rasmussen K, et al. Continuation electroconvulsive therapy vs pharmacotherapy for relapse prevention in major depression: a multisite study from the Consortium for Research in Electroconvulsive Therapy (CORE). Arch Gen Psychiatry 2006; 63: 1337-44. 